\section{Tibia vara or Blount's disease: Why an early diagnosis and treatment are important?}

\author{
Angelo V. Vasiliadis, ${ }^{1,2}$ \\ Alexandros Maris, ${ }^{3}$ \\ Srinivas Gadikoppula ${ }^{3}$
}

$1^{\text {nd }}$ Orthopaedic Department of

Papageorgiou General Hospital,

Thessaloniki, Greece; ${ }^{2}$ School of

Medicine, Aristotle University of

Thessaloniki, Thessaloniki, Greece;

${ }^{3}$ Department of Trauma and

Orthopaedics, North Middlesex

University Hospital, London, UK

\begin{abstract}
Tibia vara is an asymmetrical disorder of the proximal tibia that produces a threedimensional deformity, which was first described by Blount in 1937. A 6-year-old boy presented with a history of anterior knee pain with progressive bowing of his right leg over the last 1 year after having a fall while playing soccer. An early diagnosis and treatment of this disease can have a great functional impact and lead to very good health outcomes.
\end{abstract}

\section{Case Report}

A 6-year-old Black African boy presented in the Hospital clinic complaining for anterior knee pain with progressive bowing of his right leg that commenced to become noticeable over the last 1 year, when he fell while playing soccer. His parents reported that the knee pain was not consistent and it came and gone only during sports activities. There were no other complaints. Birth and developmental history was normal and his mother confirmed that he did not suffer from any medical condition or take any sort of medication before. The fine/gross motor skills, social, emotional and language milestones were also normal. He started walking without support at the age of 11 months. The clinical examination did not reveal any leg length discrepancy with upper: lower segment ratio of 1.15:1. He had an ideal weight (weight: $24 \mathrm{~kg}$; height: $122 \mathrm{~cm}$; BMI: $16.1 \mathrm{~kg} / \mathrm{m}^{2} ; 70^{\text {th }}$ percentile line). Plain radiograph of right leg revealed tibia vara with medial breaking and a significant depression of the medial tibial epiphysis and metaphysis (stage IV of Langenskiöld). The angle between the femoral and the tibial shaft (F-T angle) was $11^{\circ}$ and the angle between femoral condyles and the tibial shaft (FC-T angle) was $72^{\circ}$. As for the angle of the medial tibial plateau depression (MTPD angle) and the Drennan metaphyseal-diaphyseal angle, they were $80^{\circ}$ and $21^{\circ}$ respectively (Figure 1 ).

\section{Discussion}

Blount's disease is defined as an acquired growth disorder of the medial aspect of the proximal tibial physis and epiphysis resulting in progressive lower-limb deformity. Although it is also known as tibia vara, this disease usually results in a multiplanar deformity of the limb dominated by varus, procurvatum and internal rotation of the tibia. ${ }^{1}$ This clinical entity has two different main types based on the child's age: i) the infantile, which is typically noted between 2-5 years of age; and ii) the adolescent, which appears in children older than 10 years. ${ }^{2}$ The etiology of this disease is not clear, however, it is likely to be multi-factorial but surely related to mechanical overload in genetically susceptible individuals. Additionally, predisposing factors have been proposed including obesity, large stature, early walking, African-American children or a combination of the above factors. ${ }^{1,3}$ A sharp index of suspicion for Blount disease is essential in the time of the diagnosis, especially for a child older than 3 years old with excessive varus deformity focused at proximal tibia. ${ }^{3}$ Plain radiograph is a simple and useful tool to diagnose Blount's disease in patients older than 2.5 years of age. ${ }^{1}$

Early diagnosis and treatment of this disease is vital, because in the early stage of the disease, the process is reversible. The usefulness of conservative treatment with the utilization of bracing in the early stage of the disease remains debated..$^{1,4}$ It is not surprising that the unloading of the medial joint with slight overcorrection by either efficient bracing or/and an osteotomy may be beneficial in the very early stages of the disease (stage I and II of Langenskiöld). ${ }^{5}$ Previously characterized failure factors of conservative treatment with bracing include obesity (with BMI $>20 \mathrm{~kg} / \mathrm{m}^{2}$ or $/$ and $>90^{\text {th }}$ percentile line), varus thrust, age ( $>3$ years of treatment initiation), bilaterality and severe disease $(\geq$ stage III of Langenskiöld). ${ }^{1,5}$ In cases of failure of the brace treatment, the recommendation is generally a proximal tibial osteotomy, which must be performed before the $4^{\text {th }}$ year of age and only when is indicated. ${ }^{1,4}$ Siregar $^{6}$ described a new therapeutic technique in a case with stage IV of Langenskiöld, that involves multiple longitudinal osteotomies
Correspondence: Angelo V. Vasiliadis, $2^{\text {nd }}$ Orthopaedic Department of Papageorgiou General Hospital, Ring Road-N.Eukarpia, 56403 Thessaloniki, Greece.

Tel.: +30.6948402828 -

Fax: +30.2310914437.

E-mail: vasiliadis.av@gmail.com

Key words: Tibia vara; Blount's disease; diagnosis; treatment.

Acknowledgments: the authors are grateful to the patient and his parents for their cooperation in preparing this clinical image.

Contributions: AVV, AM, manuscript idea and writing; AVV, literature search; AM, data collection and/or processing; AVV, AM, SG, critical review of the manuscript, supervision and responsibility for the course of the project and the manuscript preparation.

Conflict of interest: the authors declare no potential conflict of interest.

Received for publication: 30 October 2019.

Revision received: 12 April 2020.

Accepted for publication: 6 May 2020.

This work is licensed under a Creative Commons Attribution NonCommercial 4.0 License (CC BY-NC 4.0).

(C) Copyright: the Author(s), 2020

Licensee PAGEPress, Italy

Clinics and Practice 2020; 10:1222

doi:10.4081/cp.2020.1222

over the proximal tibia without loss of cortical continuity and is called Bamboo osteotomy. The author concluded that his technique allows multi-planar correction, provides more stability and can be expected to recover faster than conventional open wedge osteotomy. ${ }^{6}$

The management of neglected cases, in patients who suffer from Blount disease of stage $\mathrm{V}$ or greater according to the Langenskiöld classification, can be a real challenge for orthopaedic surgeons and may require a combination of very demanding surgical procedure of distal femoral osteotomy with a tibial osteotomy, followed by gradual correction using an external fixator. ${ }^{1}$ Corrections of Blount's disease with multiple osteotomies in tibia, fibula and femur can be performed in multi-planar deformities, which occur in early onset and stage II to VI of Langenskiöld. ${ }^{7,8}$ Diaphyseal and distal femoral deformities can be corrected with femur valgus osteotomy with screws/plate and with medial hemi-epiphyseal staples respectively. The overall functional outcomes seem to be 


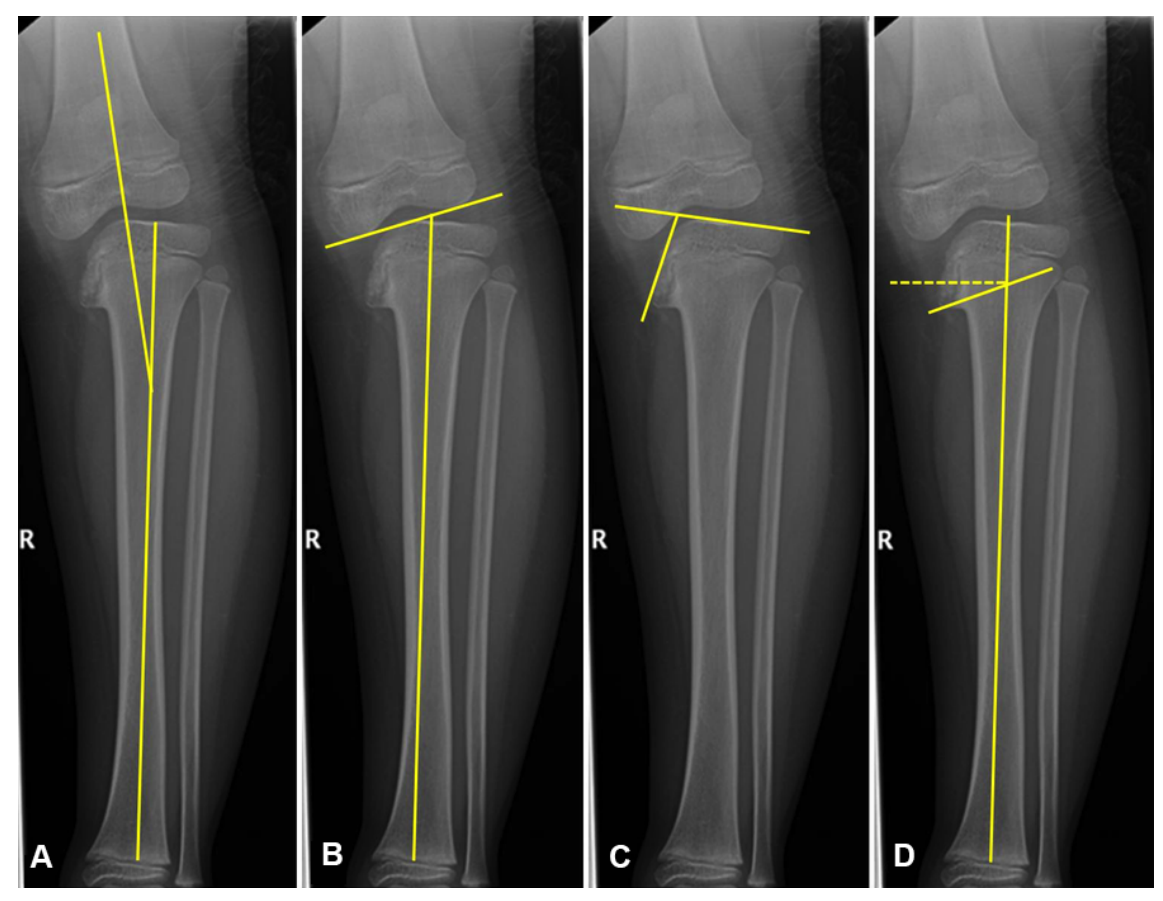

Figure 1. Angles measured on AP radiographs. The angle between the femoral and the tibial shaft (A), the angle between the femoral condyles and the tibial shaft (B), the angle of the medial tibial plateau depression (C) and the Drennan metaphyseal-diaphyseal angle (D).

good, while recurrence rates may be correlated with the patient's weight. ${ }^{7,8}$ It is proposed that only when procurvatum is greater than the varus, fibula osteotomy should be additionally suggested, increasing the risk of peroneal nerve palsy. The use of the Ilizarov and Taylor Spatial frame are modern therapeutic options that have been used to gradually correct multi-planar deformities, accompanied with the advantages of the early weight-bearing and early motion. ${ }^{1,2}$ The Taylor Spatial frame allows six-axis correction of the most complex deformities and is able to correct varus, procurvatum and internal tibial torsion simultaneously in the same frame without any special additional modification. oped and used for the treatment of Blount's disease. The best treatment for this disease, that can be accompanied with the best functional outcomes depends on the patient's age, severity of the deformity at the time of the intervention, psychosocial factors and experience of the orthopedic surgeon.

\section{References}

1. Janoyer M. Blount disease. Orthop Traumatol Surg Res 2019;105:S111-12.

2. Gkiokas A, Brilakis E. Management of neglected Blount disease using double corrective tibia osteotomy and medial plateau elevation. J Child Orthop 2012; 6:411-8.

3. Birch JG. Blount disease. J Am Acad Orthop Surg 2013;21:408-18.

4. Heflin JA, Ford S, Stevens P. Guided growth for tibia vara (Blount's disease). Medicine (Baltimore) 2016;95:e4951.

5. Sabharwal S, Sabharwal S. Treatment of infantile Blount disease: An update. J Pediatr Orthop 2017;6:S26-31.

6. Siregar PU. Bamboo osteotomy for Blount's disease. Malays Orthop J 2010;4:56-9.

7. Liu J, Cao L, Guo SF, Xue W, Chen ZX, Tai HP, He ZR, Qian YW. Melt-metaphyseal and diphyseal osteotomy for correction of infantile Blount's disease: A long-term follow-up study. Int J Clin Exp Med 2015;8:2480-3.

The initial management of Blount's disease is leg bracing for patients aged below 3 years. If the deformity is not corrected before the age of 4 years or with those who present with Langenskiöld stage III or greater, surgery may be recommended. In surgical correction of Blount's disease, metaphyseal osteotomy remains a foundational treatment option. It is obvious that many surgical techniques have been devel-
8. Edwards TA, Hughes R, Monsell F. The challenges of a comprehensive surgical approach to Blount's disease. J Child Orthop 2017;11:479-87.

9. Eidelman M, Bialik V, Katzman A. The use of the Taylor spatial frame in adolescent Blount's disease: Is fibular osteotomy necessary? J Child Orthop 2008;2:199-204. 